\title{
Tissue Engineered Airways: A Prospects Article.
}

\author{
Stephanie L. Bogan*, Gui Zhen Teoh* and Martin A. Birchall
}

\begin{abstract}
An ideal tracheal scaffold must withstand luminal collapse yet be flexible, have a sufficient degree of porosity to permit vascular and cellular ingrowth, but also be airtight and must facilitate growth of functional airway epithelium to avoid infection and aid in mucocilliary clearance. Finally, the scaffold must also be biocompatible to avoid implant rejection. Over the last 40 years, efforts to design and manufacture the airway have been undertaken worldwide but success has been limited and far apart. As a result, tracheal resection with primary repair remains the Gold Standard of care for patients presenting with airway disorders and malignancies. However, the maximum resectable length of the trachea is restricted to $30 \%$ of the total length in children or $50 \%$ in adults. Attempts to provide autologous grafts for human application have also been disappointing for a host of different reasons, including lack of implant integration, insufficient donor organs and poor mechanical strength resulting in an unmet clinical need. The two main approaches researchers have taken to address this issue have been the development of synthetic scaffolds and the use of decellularised organs. To date, a number of different decellularisation techniques and a variety of materials, including polyglycolic acid (PGA) and nanocomposite polymers have been explored. The findings thus far have shown great promise, however there remain a significant number of caveats accompanying each approach. That being said, the possibilities presented by these two approaches could be combined to produce a highly successful, clinically viable hybrid scaffold. This article aims to highlight advances in airway tissue engineering and provide an overview of areas to explore and utilise in accomplishing the aim of a developing an ideal tracheal prosthesis.
\end{abstract}

\section{Introduction}

Disorders of the trachea can lead to serious health complications, significantly reducing the quality of life for patients. These vary but include tracheal carcinoma, tracheo-esophageal fistulae, stenosis, tracheobronchial malacia and injury, or the ingestion of harmful substances. Existing treatment options are limited; currently the Gold Standard treatment is tracheal resection with a primary reconstruction to restore continuity. Other therapies include aortopexy, tracheal stenting, or slide tracheoplasty, but these have mixed levels of success. To date, there is no clinically viable option available for patients with long segmental airway disorders. In adults, only half of the tracheal length, approximately $6 \mathrm{~cm}$, can be successfully treated by resection, and even less in children where only one third of the length of the trachea can be reconstructed ${ }^{1,2}$.

Alternative options for trachea replacement are being developed, including autografts, allografts, and prosthetics, or a combination of these; but they have limitations including inflammation, infection, improper sizing, and a failure to effectively mimic the physiological properties of the original tissue ${ }^{1-8}$. With the advancements made in tissue engineering (TE) in the last 40 years ${ }^{4,9}$, researchers are focusing on utilising a tracheal scaffold made from a suitable synthetic polymer or a cadaveric allotransplant. To properly engineer a functional implant one must understand the physiology of the trachea (Figure 1). 


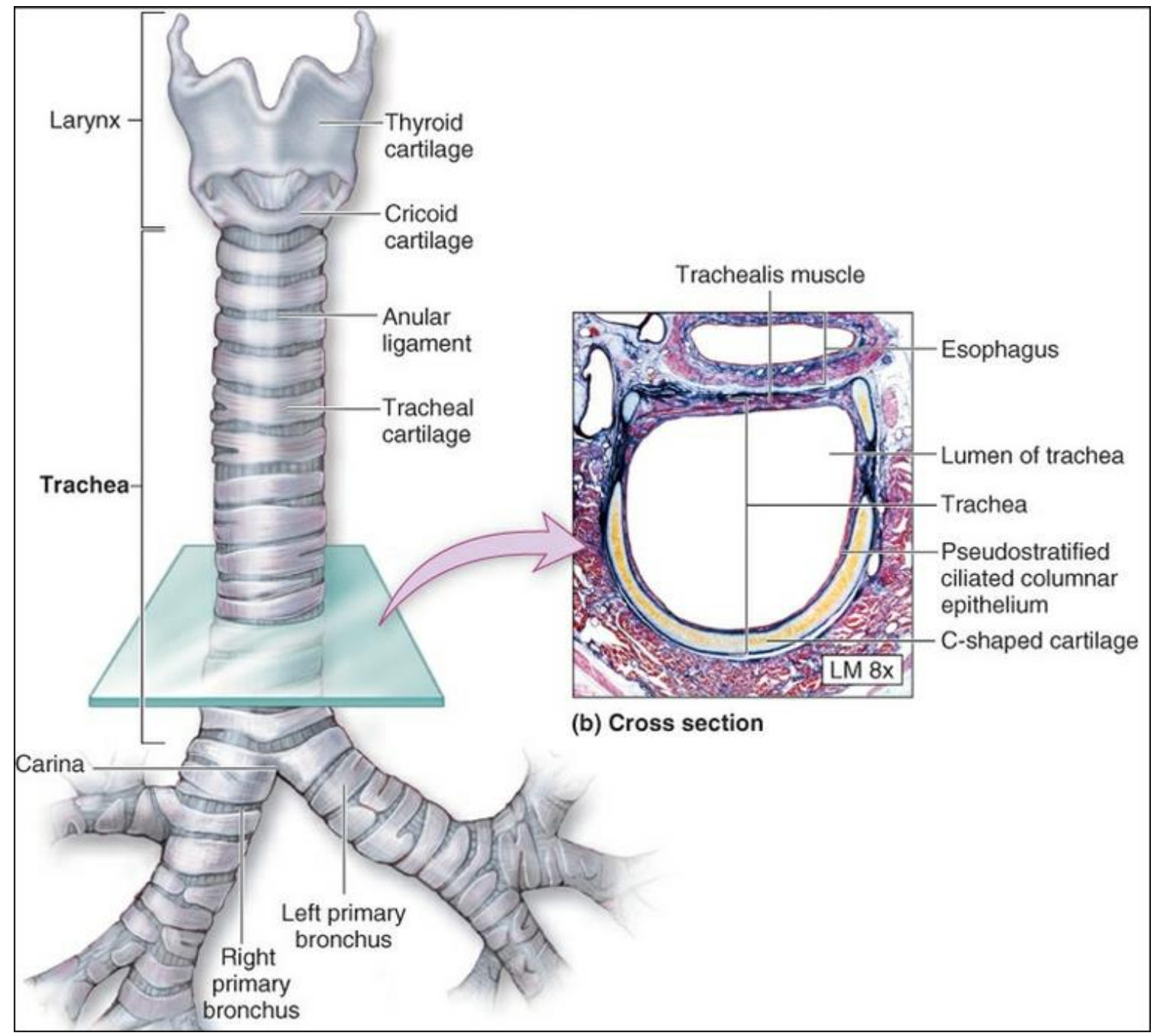

Figure 1: Anatomy of the human trachea.

The trachea is a vascularised hollow tube that forms the airway passage between the larynx and the lungs. Structurally, it is composed of layers of connective tissue and smooth muscle, supported by U-shaped rings of hyaline cartilage that hold it open. Internally it is lined with pseudostratified columnar epithelium, with ciliated, brush, basal, and secretory cells on a basement membrane ${ }^{10}$. The trachea develops from the respiratory diverticulum. In the third to fourth week of life, the hepatic primordium migrates from the respiratory primordium, allowing the respiratory primordium to dilate and bifurcate ventrocaudally into lung buds. The lung buds give rise to the trachea, infra- glottis, and the glottic opening. By week 8 , the mesenchymal rudiments of the tracheal cartilages are present. During the next 2 weeks, the cartilages develop into fibro-elastic tissue, and smooth muscle is incorporated into the trachea. 


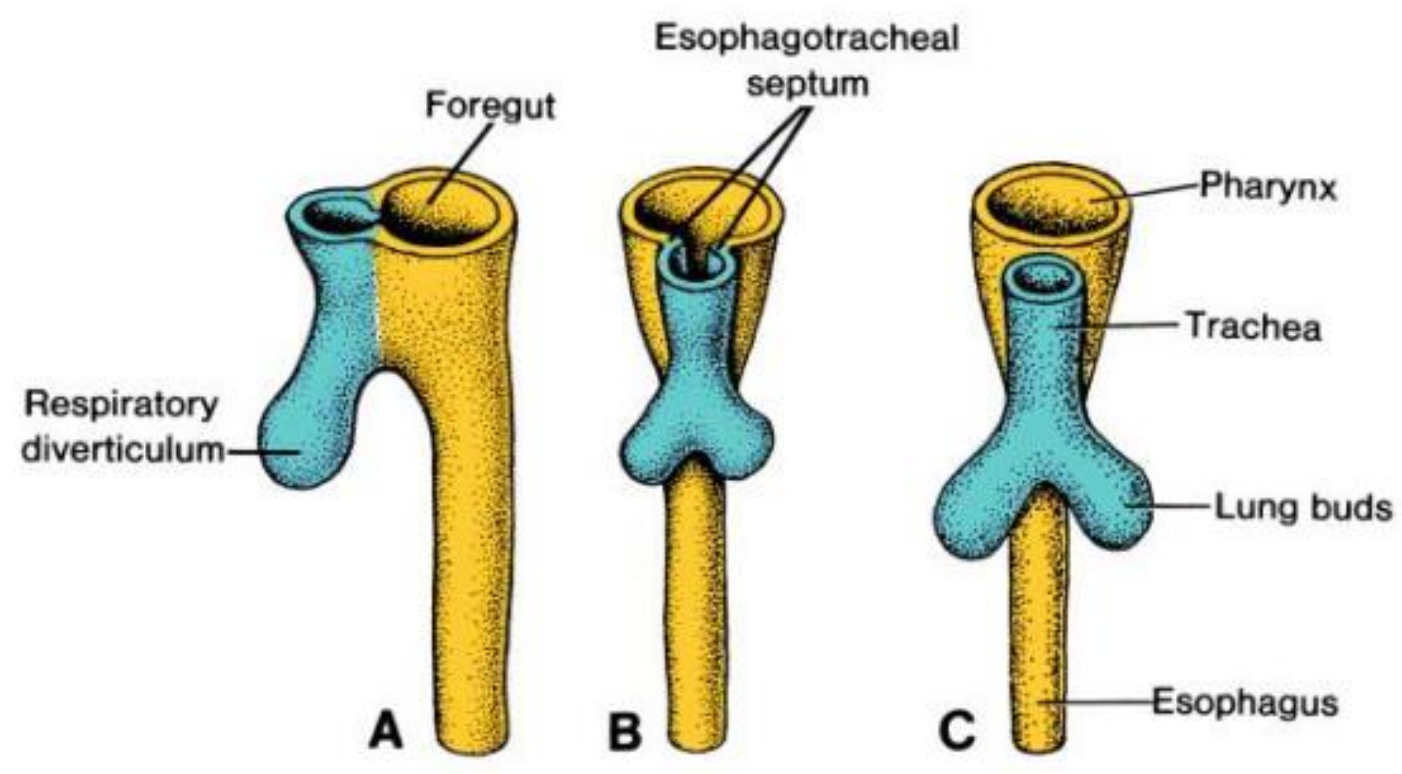

Figure 2: Development of the trachea. A. Lateral view at the end of week 3; B-C. Ventral view of development at week 4.

The view is that the tissue engineered airway will replace the damaged organ, mimic the native extracellular matrix (ECM) in structure and function, while also providing support and encouraging cell attachment, differentiation, and proliferation ${ }^{11}$. This technique was originally pioneered by Langer and Vacanti ${ }^{12}$ who showed that scaffold composition is a key feature, and can accelerate tissue regeneration even without cells or growth factors. Scaffolds may be biological, as a decellurised allotransplant, or composed of a synthetic material, which can be non-biodegradable or biodegradable. Synthetic scaffolds have been studied in various forms including polyethylene glycol-based hydrogel, polylactic/glycolic acid (PLGA), poly(e-caprolactone) (PCL), polyester-urethane, gelatin sponge, Marlex mesh and other modified forms (see table 1). While many of these innovative treatments could potentially be utilised in adults, the treatment options for children are limited as the level of complexity increases due to variations in the size of the trachea, and changes over time due to growth. Here the focus is to review the application of decellularised scaffolds and synthetic and biodegradable polymers in tissue engineered airways, highlighting cellular interaction, and clinical applications. 


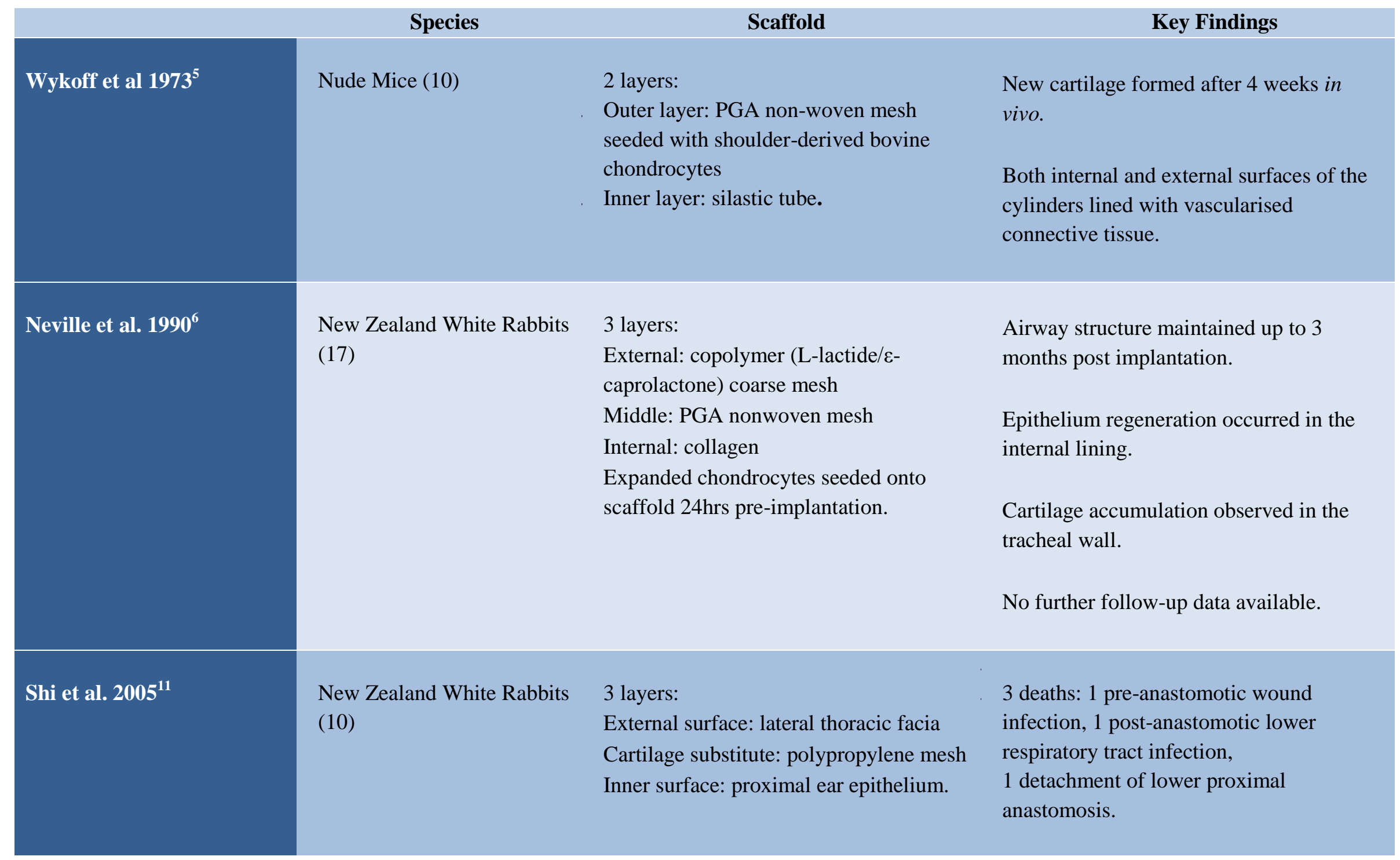




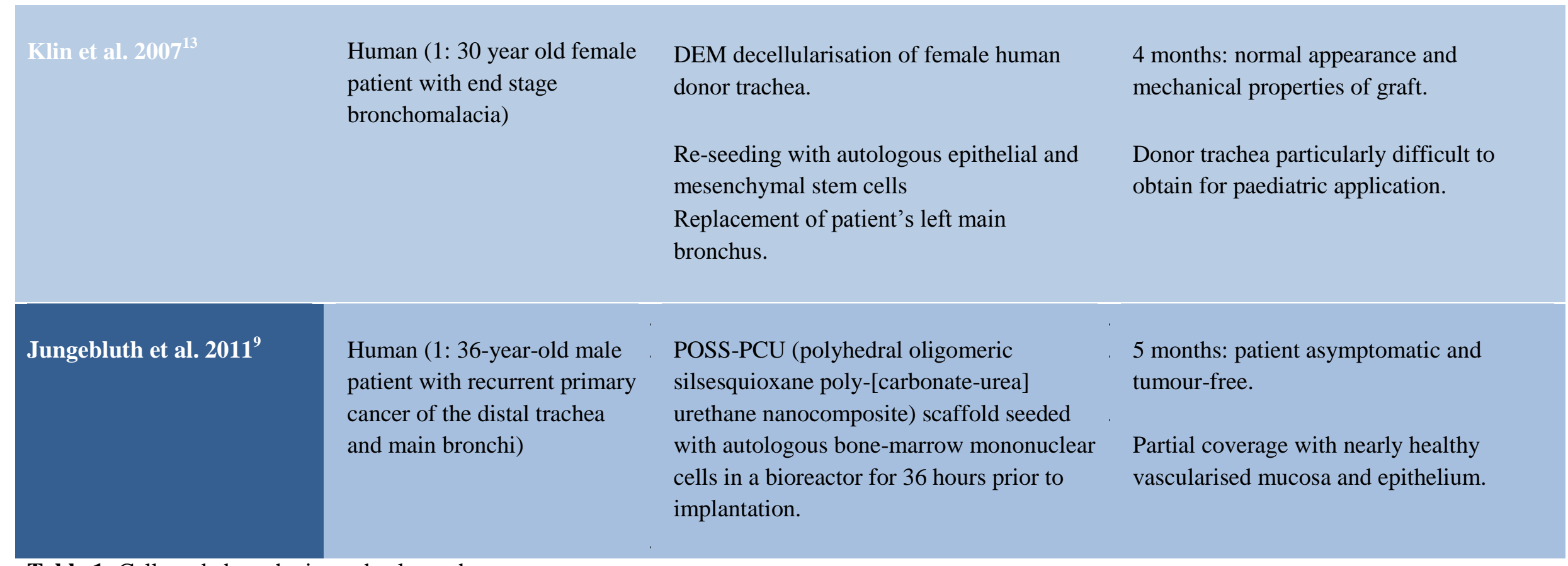

Table 1: Cell seeded synthetic tracheal prostheses 


\section{Scaffolds}

Tissue engineered scaffolds are designed to act as a supporting structure; maintain an open airway and mimic the biological and mechanical function of the native ECM; providing a template for the stem cells until they have proliferated, regenerated, and stabilised the damaged area ${ }^{9}$ (Figure 3).

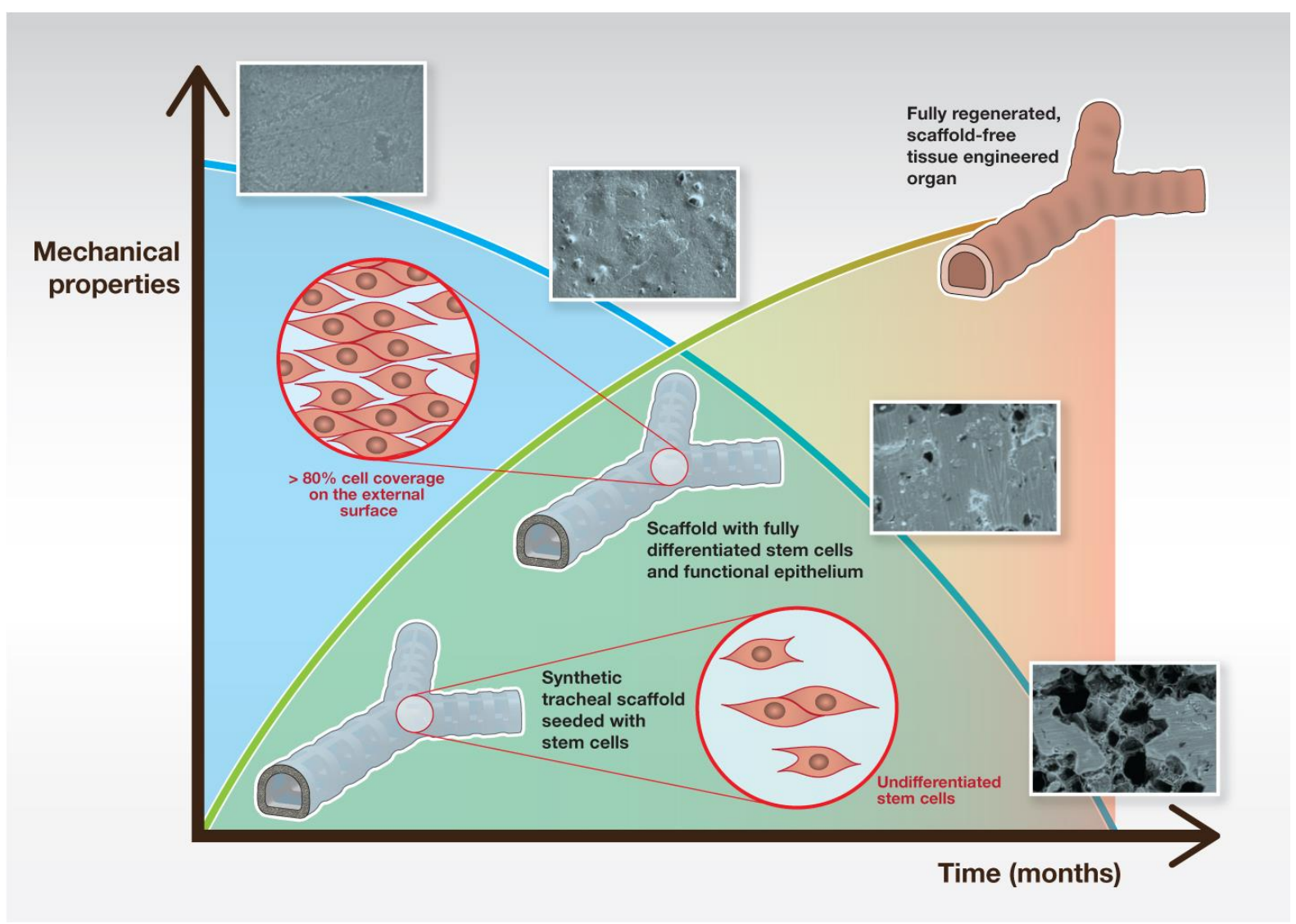

Figure 3: Schematic representation of the relationship between degradable scaffolds and tissue regeneration

Most importantly it must be nontoxic, laterally rigid to prevent collapse, while also being flexible to accommodate the necks natural movements. Flow of air into the trachea makes it prone to airborne infections such as bacterial and viral infections ${ }^{13}$. In native tissue, cilia provide a barrier and a mechanism for the expulsion of particulates. To mimic this action vascularization is needed at the air-tissue interface. A porous scaffold allows cell-polymer interactions for the ingrowth of host connective tissue ${ }^{6,7}$. It should also be biocompatible in terms of cell type, function, longevity, and immunogenicity. A scaffold that does not initiate a secondary immune response would eliminate the risk of implant rejection as well as the need for life-long immunosuppression, which is unsuitable in patients with cancers of the trachea. For degradable polymers the degradation products must be biologically safe. The scaffold also needs to degrade at an appropriate rate in relation to the regeneration of tissue to prevent structural collapse of the trachea.

The type of scaffold is still an on-going debate but methods for tracheal replacement include the use of autologous tissue $^{14}$ autografts $^{15}$, allografts ${ }^{7}$ prosthetic materials ${ }^{4,16,17}$ or a combination of these approaches ${ }^{1,18}$. These works have met with limited success due to stenosis, immunological rejection, bacterial infections, graft migration and material failure ${ }^{11}$. Tissue engineered polymeric implants are preferable as they do not raise a 
substantial immune response, which can often be seen in allografts. Despite this TE tracheal transplants have already been carried out clinically in a few compassionate cases ${ }^{7,13,19-21}$. None of the current scaffold approaches have the potential to grow with the patient; therefore, sustainability is limited in growing children ${ }^{3}$. There are three areas of focus for scaffolds used in airway tissue engineering: allogenic decellurised tissue, and biodegradable, or non-biodegradable synthetic scaffolds.

\subsection{Decellularised Scaffolds}

Decellularised scaffolds are currently the most widely used TE tracheal implant in the clinical setting. To create a decellularised scaffold a donor trachea is harvested from a cadaver, subjected to numerous washing cycles to remove donor cells via detergents, salts, enzymes, and/or physical means ${ }^{22,23}$ and is then recellularised with the patient's own cells. This gives a TE trachea good biomechanical properties, functional extracellular matrix (ECM), and removes the possibility of an immune response by eliminating major histocompatibility complexes class $\mathrm{I}$ and $\mathrm{II}^{24}$. For the scaffold to fully integrate it must have a healthy ECM composed of proteins, collagen subunits, proteoglycans and glycoproteins; all of which the decellularisation process retains, maintaining the tissues ability to develop and grow, and also removes immune responsiveness ${ }^{25}$.

Decellularised tissue engineered tracheas have been investigated in a number of studies to date, with varied rates of success ${ }^{8,25-27}$. While good epithelialisation was observed in many, the grafts were structurally unstable leading to airway obstruction and subsequent collapse. The first in human tissue-engineered tracheal replacement was performed in 2008 , on a 30 -year old female patient with end-stage bronchomalacia ${ }^{13}$. A donor trachea was decellurised via the detergent-enzymatic method ${ }^{22}$ and seeded with epithelial cells and mesenchymal stem cell-derived chondrocytes from the recipient. The graft was structurally sound with good rates of revascularisation, epithelialsation and no immunological response or serological signs of rejection. On follow-up 5 years later the tissue-engineered trachea itself was patent, well vascularised, completely recellularised with respiratory epithelium, and had normal ciliary function and mucus clearance ${ }^{28}$. However, patency of the lumen was suboptimal with the graft requiring dilatation and stenting intermittently.

Since this case, there have been several other clinical cases performed worldwide but very few involved children receiving a tissue engineered tracheal transplant. One case in 2012 saw the use of a decellularised donor trachea, implanted into a 10-year-old boy to repair a $7 \mathrm{~cm}$ section of damaged trachea, due to long-segment congenital tracheal stenosis and pulmonary sling. No seeding with epithelial cells, mesenchymal stem cells (MSC), or chondrocyte differentiation occurred before the surgery due to the immediacy of the procedure. Instead an intraoperative procedure was used; prior to implantation the donor trachea was saturated with a cell suspension of haematopoietic and MSCs and also human recombinant erythropoietin. Granulocyte-colony stimulating factor (G-CSF) and transforming growth factor beta (TGF-B) were injected to the tracheal rings to aid MSC proliferation and recruitment, chondrocyte differentiation, and angiogenesis. Temporary stenting was required following surgery, while bronchoscopy and balloon dilation were carried out regularly to clear mucus build-up. Nevertheless, the clinical course was good. At 6 months post-op, the airway was patent and stable, balloon dilation was still needed, but at 18 months post-surgery the child was healthy and fit with no signs of complication $^{19}$. 
Additionally, a case series of nine adult and paediatric patients were reported in a review by Badylak et al. ${ }^{29}$ in 2012 which stated that there were no graft related mortalities 12-42 months following surgery, however partial graft collapse had occurred in some instances. Unfortunately, these cases have not yet been reported or followed up in a formal scientific paper, making it difficult to surmise the outcomes. While decellularised donor implants have been successful in certain compassionate cases, the method has not been optimised or clinically trialed yet. In the future if a standard framework for this procedure can be identified and tested it is possible these could be used clinically, but will still be limited by the shortage of donor tissue.

\subsection{Synthetic Scaffolds}

In tissue engineering, one of the most debated issues is the choice of synthetic scaffold. There are numerous options for a synthetic scaffold as previously mentioned, but these are generally composed of modified polymers, often in combination with biological elements. Studies have shown that inserting chondrocytes for cartilage growth in natural materials like gels or sponges, including alginate, collagen and agarose, can help to promote collagen type II and proteoglycan synthesis ${ }^{16}$. Omori et $\mathrm{al}^{30}$ developed a collagen-conjugated prosthesis for tracheal replacement composed of polypropylene Marlex mesh, a spiral ring frame, and collagenous sponge extracted from porcine skin. This scaffold was applied to repair the larynx and trachea in 4 adult patients. Postoperative results were positive, and even though epithelialisation on the luminal surface took some time to develop, follow-up studies reported good epithelisation and no signs of graft rejection after three years.

A novel synthetic nanocomposite polymer reseeded with autologous mononuclear cells was used to create the worlds' first completely synthetic tracheal transplant in $2011^{31}$. The POSS-PCU nanocomposite material is composed of a polycarbonate (urea) urethane soft segment (PCU) and a polyhedral oligomeric silsequioxane (POSS) cage attached as a pendant group. This non-biodegradable material is biocompatible, nontoxic, and elicits a low inflammatory response in vivo ${ }^{32}$. The recipient, a 36 year-old male, suffered from recurrent primary cancer of the distal trachea and main bronchi. As resection was unsuitable in this case, a novel bioartificial nanocomposite was designed to replace the section of trachea affected. The initial surgical outcome was encouraging, however biopsy samples showed the presence of necrotic connective tissue due to fungal infection. Nevertheless, 5 months after transplantation the patient was asymptomatic, did not show signs of rejection, had a patent airway and the mucosa was vascularized with a near-normal epithelium.

While these materials provide researchers with a highly modifiable graft that can be customised according to the requirements of the patient, there are still major associated risks. The synthetic materials can be poorly incorporated by native tissues; resulting in complications such as graft migration, stenosis, infection and the formation of granulation tissue at the anastomosis site.

\subsection{Biodegradable Synthetic Scaffolds}

For paediatric applications, the choice of scaffold is a key consideration. Tracheal defects affecting children include congenital and pediatric lesions, tracheal stenosis, tracheoesophageal fistulae, complete tracheal rings, tracheomalacia, laryngotracheoesophageal cleft, and damage due to prolonged intubation and tracheal trauma ${ }^{33}$. The treatment options available to children are even more limited in comparison to adults as the maximum resectable length of the trachea is limited to $30 \%$ due to the amount of tissue available. Thus, there is currently no viable tracheal replacement for children. For paediatrics, the use of a biodegradable scaffold that replaces the 
damaged tracheal section and supports tissue modification, cellular migration and proliferation, while also degrading at a suitable rate during growth would be highly desirable, as it would eliminate the need for recurrent surgeries. Polymers can degrade in two distinct ways; via surface or bulk degradation. In surface degradation the material is eroded layer by layer, reducing in thickness whilst maintaining the original shape, however in bulk degradation the polymer is lost through disintegration, weakening the device at unspecified points across the surface of the scaffold. This distinction is important when thinking about the structural integrity of the implant, because if it degrades at a rate that is too rapid, there is an increased risk of luminal collapse. As the polymer erodes, the cells must also be able to withstand the changeable environment. Equally, the degradation products must not be toxic, or initiate an immune response. The rate of degradation is malleable as it is dependent on the type of chemical bonds in the material, $\mathrm{pH}$ of the environment, polymer composition, and water uptake ${ }^{34}$.

Synthetic scaffolds hold much promise for future directions but problems such as poor biocompatibility, graft migration, and insufficient integration with the native tissue, remain unresolved. Furthermore, biodegradable scaffolds also have unique complications to overcome, like the release of harmful degradation products and loss of mechanical properties over time. The majority of biodegradable polymers being studied for clinical application are from the polyester family. These are based on lactic and glycolic acids that degrade in vivo via hydrolysis of the ester backbone, producing clean by-products that can be easily excreted by the body. Poly(glycolic acid) (PGA), poly (lactic acid) (PLA), Poly(caprolactone) (PCL) and their copolymers are the most widely used biodegradable polymers in tissue engineering ${ }^{35}$.

\section{Biodegradable Tissue Engineered Airways}

Poly(glycolic acid) (PGA) has excellent properties for use in tissue engineering. Its degradation product, glycolic acid, is converted to carbon dioxide and water; these can then be excreted through the lungs or kidneys, making it non-toxic and non-immunogenic. As a material, PGA serves as a conducive environment for cell growth, its degradation rate can be adapted, and porosity modified to encourage ECM formation and vascularisation through cell-polymer interactions ${ }^{36}$. Since the $1970 \mathrm{~s}$, the main use of PGA has been to produce resorbable sutures, but it is now being used in mesh-form for tissue engineering purposes ${ }^{35}$.

Polylactic acid (PLA) is commonly used in scaffolds, drug delivery systems, bone fixation devices such as screws and plates, surgical sutures, and meshes. PLA's degradation product is lactic acid, which naturally occurs in the body. Due to the similarities between PLA and PGA, scaffolds are often formed from a copolymer of these. Since PLA is more hydrophobic than PGA, this changes the rate of degradation and mechanical stability of the scaffold as the ester bonds in PLA are more resistant to hydrolysis ${ }^{35}$. When degrading, PLA and PGA can produce high concentrations of lactic and glycolic acids, these can negatively impact on the local environment due to the acidic content.

With a degradation time between two to three years, Poly(caprolactone) (PCL) is useful as a base polymer for long-term biodegradable implants, as it degrades at a slower rate than PLA or PGA ${ }^{37}$. Polyurethanes were once unsuitable for use in vivo due to the toxicity of their degradation product (2,4-diaminotoluene). Since then, biodegradable polymers have been modified such that degradation products are now non-toxic ${ }^{38}$. The incorporation of polyhedral oligomeric silsesquioxane (POSS) into poly ( $\mathcal{E}$-caprolactone) (PCL) has improved the polymers mechanical properties further, preventing the ester bonds in PCL from degrading through 
enzymatic attack to the same extent. The properties of these nanocomposite materials lend themselves to the development of a multitude of scaffolds and organs. Biodegradable polymers have been used to create vascular grafts $^{39}$, resorbable sutures, drug delivery systems, orthopaedic fixtures ${ }^{35}$, artificial skin, even bone substitutes ${ }^{40}$. One of the most applicable uses for biodegradable polymers has been to create resorbable stents. Stents are currently manufactured from non-resorbable materials, like plastic and metal. These lead to patient discomfort, and significant clinical complications such as migration, haemorrhage, infection, stenosis, and tissue granulation. Biodegradable stents have already been developed for oesophageal, intestinal, urethral, biliary duct, vascular stenosis and more recently for airway stenosis, therefore could be modified to produce larger constructs like tracheal scaffolds ${ }^{41}$.

In paediatric cases the use of biodegradable scaffolds, rather than an allotransplant, is also preferable for tracheal replacement, which becomes obvious when the following factors are considered. The use of tracheal allotransplants has had a certain amount of success clinically for both adults and children, but the sustainability of such implants is limited. The availability of donor tissue is a rate-limiting step in the process, one that also requires the tissue to be compatible in terms of size and suitability. The long-term outcomes from the use of decellurised donor scaffolds are not known yet, and it remains to be seen if the detergent-enzymatic method (DEM) removes all potential immune responses, or if the stringent chemical processes raise any complications in vivo. DEM is not a quick process; to fully remove the MHC antigens class I and II along with the DNA and leukocytes requires 25 washing cycles. Donor scaffolds are not available on demand and the time taken to prepare a scaffold, even when expedited, requires 5 weeks $^{42}$. For use in paediatric cases finding a size match adds another level of complexity. A biodegradable scaffold would provide an alternative, sustainable option, which can degrade as the child grows and the native tissue regenerates. If the scaffold could be optimised it could mean being able to repair a defect in one procedure, a feature allotransplants cannot currently offer.

\section{Cell Interactions}

\subsection{Cell Selection}

Two cell types essential for the success of a synthetic trachea are epithelial cells to line the airway, and chondrocytes to form new tracheal cartilage. Cell populations that make up this ciliated pseudostratified columnar epithelium include ciliated, goblet, and basal cells. As the trachea is exposed to the external environment through the passage of air, respiratory epithelium provides essential protection and maintains homeostasis. This cell network produces mucosal layers to trap particulates and pathogens, clear mucus, maintain the water/ion balance, and sustain a healthy epithelium throughout the repair and regeneration process. Chondrocytes produce and conserve the extracellular matrix of cartilage. The main sources for chondrocytes are auricular, nasal, tracheal, and costal cartilage. The U-shaped rings of the trachea are formed from hyaline cartilage, which is only present in the joints, the respiratory tract, and the immature skeleton. The distinction between the types of cartilage (hyaline, elastic and fibrocartilage) can be problematic when sourcing and culturing chondrocytes for a specific purpose. Chondrocytes have proven more difficult to harvest and grow than epithelial cells. To address this issue, bone marrow mesenchymal stem cells (MSCs) can be employed and subsequently differentiated into chondrocytes as well as epithelial cells. Over time, chondrocytes cultured in vitro, irrespective of their source, change their gene expression and effectively dediffrentiate. To account for this 
behaviour, chondrocytes are often seeded onto or within gels like agarose or collagen, as the three dimensional environment appears to better support their differentiation into hyaline cartilage ${ }^{3,24,43}$.

Cell sources available for use in tissue engineering include induced pluripotent stem cells, adult stem and progenitor cells, and lineage-committed and differentiated adult tissue cells. Cell choice is dependent on availability, expansion capacity, accessibility, and its potential for tumorgenicity through differentiation.

Embryonic stem cells are the undifferentiated inner mass cells of a human embryo. As the earliest pluripotent stem cells, they have the potential to differentiate into multiple lineages. Alternatively, cells can be sourced from foetal, umbilical cord, or placental tissue. These cell populations also have a high expansion capacity but the cell fates are already somewhat defined and long-term they have the tendency to become cancerous. While they have unrivaled potential, there are tight ethical regulations surrounding the use of embryonic or foetal stem cells making them unsuitable for regular use in the seeding of tracheal scaffolds.

Induced pluripotent stem cells (iPSCs) generated from adult cell populations are an attractive alternative to embryonic or foetal stem cells. These iPSCs exhibit the same morphology, expansion and differentiation properties, and also express the same cell marker genes as embryonic stem cells. Adult somatic cells are essentially reset to progenitor cells, driven by a series of transcription factors ${ }^{44}$. However, this technique is yet to be refined as iPSCs have failed to fully commit to their reprogrammed cell lineage and have a tendency for tumorgenicity. Techniques are still improving for creating iPSCs and there is great potential for them to be a valuable source of stem cells in the future.

Adult stem cells comprise somatic cells with the ability to self renew. These can be derived from many adult tissues including adipose tissue, bone marrow, and peripheral blood. The use of an autologous stem cell population is a more viable choice when repopulating any tracheal implant, as immune matching reduces the probability of graft failure and rejection. However, harvesting these cells from the patient may prove difficult as this can be quite an invasive procedure. Though some adult stem cells have failed to proliferate fully when trialed in animal models, bone marrow mesenchymal stem cells are used regularly in clinical applications, including for tracheal scaffold reseeding. Autologous MSCs are easily accessible from the bone marrow, have no potential for immunogenicity, have a much lower incidence of tumorgenicity, and expansion rates are favourable. MSCs have the ability to differentiate into various cell types in vitro and are also involved in tissue repair. This makes them an excellent candidate for tissue engineered airways, as MSCs have been shown to differentiate into endothelial lineages ${ }^{23}$.

Fibroblasts synthesise the extracellular matrix and collagen network that forms the basic structure for all tissues. They play a key role in epithelial-mesenchymal cell interactions and have been shown to support epithelial and mesenchymal cell growth through the reconstruction of the basement membrane ${ }^{45}$. Fibroblasts also improve cell migration, proliferation, and differentiation into ciliated, goblet and basal cells, allowing for the reconstruction of a pseudostratified ciliated epithelium similar to a native epithelium. ${ }^{45}$ They've also been shown to increase the rate of epithelial cell growth, although the source of fibroblasts is a determining factor; gingival fibroblasts were indicated to be better than nasal and dermal fibroblasts ${ }^{45,46}$. The range of cells applicable to airway tissue engineering suggests that the development of a seeded constructs is a viable option, therefore this is a promising experimental approach. 


\section{Experimental approach}

In addition to decellularised and seeded scaffolds, 3D printing and bioprinting have become highly adaptable techniques for creating bespoke tissue engineered scaffolds. This technique permits the production of 3D solid scaffolds of any shape from digital images i.e. CT scans, giving a high level of reproducibility and control over the material properties of the scaffold, such as porosity and surface roughness. Advancements have been made with the development of bioprinting; allowing for both the printing of a support structure on which the cells can proliferate, and the incorporation of cell populations and biological cues into the ink, resulting in a completely cohesive scaffold.

The methods for 3D printing vary; they include inkjet, laser-induced forward transfer, microextrusion or stereolithography and projection pattern formats ${ }^{47}$. Each method comes with its own pros and cons, such as mechanical stress, cost, clogging, or limited material use. One of the main benefits in 3D printing scaffolds is the accuracy and reproducibility compared to traditional procedures, so material printability and printer method must be carefully considered. However, a key notation is that despite the advantages, the issue of selecting a suitable printing material to mimic the native tissue remains. Natural materials have an inferior structural integrity in comparison to the synthetic materials, but do provide a superior biocompatible environment. The opposite can be said for synthetic polymers, which show higher mechanical strength, but have a limited degree of biocompatibility. For this reason the combination of natural and synthetic materials into a hybrid scaffold would result in a prosthesis which satisfies the desired parameters.

The choice of cells is dependent on the target application; in this case epithelial cells and mesenchymal cells to generate airway epithelium and cartilage. However, another selection factor to consider is the robustness of the cells as they have to withstand stresses from the bioprinting process. Encapsulating cells in hydrogels creates a tissue-like environment increasing viability, which is often is often affected in bioprinting. A 3D printed PCL scaffold coated with mesenchymal stem cells seeded in fibrin was tested for the repair of partial tracheal defects in vivo ${ }^{48}$. The 3D printed scaffold integrated well with the native tissue. The reconstructed trachea was successfully covered with regenerated respiratory epithelium, neo-cartilage formed and minimal granulation tissue was seen at suture points.

The potential of bioprinting is undeniable; however as a relatively new approach for the development of tissue engineered scaffolds, refinements are needed in printing methods, ink compositions and scaffold properties.

\section{Conclusion}

After decades of research, there now appear to be promising avenues to explore in order to successfully tissue engineer the airway. As highlighted in this article, published research suggests that a promising approach may be to decellularise a cadaveric trachea and recellularise it with the patient's own cells ${ }^{24}$. This option has been applied clinically, with successful outcomes recorded from patient reports ${ }^{13,19}$. However, most published followup papers report weak mechanical properties, at least for the initial months post-implantation, with most cases requiring stenting to maintain patency ${ }^{19,28,29}$. Another issue with this approach is that it is not time efficient as the duration required find a donor and decellularise the scaffold may be problematic, specifically for cancer and trauma patients where a rapid replacement may be required. Additionally, a standard decellularisation protocol must be vigorously validated to prepare for potential variance between donor tissues. Turning to synthetic scaffolds appears to offer some theoretical advantages over scaffolds of biologic origin. These include the lack 
of an allorejection response rendering immunosuppressive therapy unnecessary, the potential to be tailor-made and/or 3D printed to each individual patient's specific requirements and dimensions and a high degree of malleability allowing for good control of the strength, microstructure, degradation rate and porosity of the scaffold. These scaffolds may be 'off the shelf' accessible for urgent cases, without waiting for donor tissue. High-throughput production can also be tightly regulated and the constructs sterilised with ease. The disadvantages of this approach, on the other hand, include mechanical mismatch and poor integration resulting in formation of granulation tissue at the anastomosis sites. With advances in 3D bioprinting technology, the combination of synthetic materials and printable biological components appears to be feasible and the most rational approach to achieving success in airway tissue engineering within the next decade. 


\section{References}

1. Hong HJ, Lee JS, Choi JW, Min B-H, Lee H-B, Kim C-H. Transplantation of autologous chondrocytes seeded on a fibrin/hyaluronan composite gel into tracheal cartilage defects in rabbits: preliminary results. Artif Organs. 2012;36(11):998-1006. doi:10.1111/j.1525-1594.2012.01486.x.

2. Kojima K, Vacanti CA. Tissue engineering in the trachea. Anat Rec (Hoboken). 2014;297(1):44-50. doi:10.1002/ar.22799.

3. Kojima K, Bonassar LJ, Roy AK, Vacanti CA, Cortiella J. Autologous tissue-engineered trachea with sheep nasal chondrocytes. J Thorac Cardiovasc Surg. 2002;123(6):1177-1184. doi:10.1067/mtc.2002.121161.

4. Grillo HC. Tracheal replacement: a critical review. Ann Thorac Surg. 2002;73(6):1995-2004. doi:10.1016/S0003-4975(02)03564-6.

5. Kushibe K, Tojo T, Sakaguchi H, et al. Effects of warm ischemia and cryopreservation on cartilage viability of tracheal allografts. Ann Thorac Surg. 2000;70(6):1876-1879. doi:10.1016/S00034975(00)01854-3.

6. ten Hallers EJO, Rakhorst G, Marres HAM, et al. Animal models for tracheal research. Biomaterials. 2004;25(9):1533-1543. http://www.ncbi.nlm.nih.gov/pubmed/14697856. Accessed January 4, 2016.

7. Delaere P, Vranckx J, Verleden G, De Leyn P, Van Raemdonck D. Tracheal allotransplantation after withdrawal of immunosuppressive therapy. N Engl J Med. 2010;362(2):138-145. doi:10.1056/NEJMoa0810653.

8. Baiguera S, Jungebluth P, Burns A, et al. Tissue engineered human tracheas for in vivo implantation. Biomaterials. 2010;31(34):8931-8938. doi:10.1016/j.biomaterials.2010.08.005.

9. Bonassar LJ, Vacanti CA. Tissue engineering: The first decade and beyond. J Cell Biochem. 1998;72(S30-31):297-303. doi:10.1002/(SICI)1097-4644(1998)72:30/31+<297::AID-JCB36>3.0.CO;26.

10. Roomans GM. Tissue engineering and the use of stem/progenitor cells for airway epithelium repair. Eur Cell Mater. 2010;19:284-299. http://www.ncbi.nlm.nih.gov/pubmed/20571996. Accessed January 4, 2016.

11. Clark ES, Best C, Onwuka E, et al. Effect of cell seeding on neotissue formation in a tissue engineered trachea. J Pediatr Surg. 2015. doi:10.1016/j.jpedsurg.2015.10.008.

12. Vacanti JP, Langer R. Tissue engineering: the design and fabrication of living replacement devices for surgical reconstruction and transplantation. Lancet. 1999;354:S32-S34. doi:10.1016/S01406736(99)90247-7.

13. Macchiarini P, Jungebluth $\mathrm{P}$, Go $\mathrm{T}$, et al. Clinical transplantation of a tissue-engineered airway. Lancet. 2008;372(9655):2023-2030. doi:10.1016/S0140-6736(08)61598-6.

14. Martinod E, Seguin A, Pfeuty K, et al. Long-term evaluation of the replacement of the trachea with an autologous aortic graft. Ann Thorac Surg. 2003;75(5):1572-1578. doi:10.1016/S0003-4975(03)00120-6.

15. Backer CL, Mavroudis C, Dunham ME, Holinger LD. Repair of congenital tracheal stenosis with a free tracheal autograft. J Thorac Cardiovasc Surg. 1998;115(4):869-874. doi:10.1016/S00225223(98)70368-X.

16. Crowley C, Birchall M, Seifalian AM. Trachea transplantation: from laboratory to patient. J Tissue Eng Regen Med. 2015;9(4):357-367. doi:10.1002/term.1847.

17. Teoh GZ, Crowley C, Birchall MA, Seifalian AM. Development of resorbable nanocomposite tracheal and bronchial scaffolds for paediatric applications. - PubMed - NCBI. British Journal of Surgery. doi:10.1002/bjs.9700.

18. Tada Y, Suzuki T, Takezawa T, et al. Regeneration of Tracheal Epithelium Utilizing a Novel Bipotential Collagen Scaffold. Ann Otol Rhinol Laryngol. 2008;117(5):359-365. doi:10.1177/000348940811700506.

19. Elliott MJ, De Coppi P, Speggiorin S, et al. Stem-cell-based, tissue engineered tracheal replacement in a child: a 2-year follow-up study. Lancet (London, England). 2012;380(9846):994-1000. doi:10.1016/S0140-6736(12)60737-5.

20. Olson JL, Atala A, Yoo JJ. Tissue engineering: current strategies and future directions. Chonnam Med J. 2011;47(1):1-13. doi:10.4068/cmj.2011.47.1.1.

21. Jungebluth P, Moll G, Baiguera S, Macchiarini P. Tissue-Engineered Airway: A Regenerative Solution. Clin Pharmacol Ther. 2012;91(1):81-93. doi:10.1038/clpt.2011.270.

22. Conconi MT, De Coppi P, Di Liddo R, et al. Tracheal matrices, obtained by a detergent-enzymatic method, support in vitro the adhesion of chondrocytes and tracheal epithelial cells. Transpl Int. 2005;18(6):727-734. doi:10.1111/j.1432-2277.2005.00082.x.

23. Scarrit ME. A review of cellularization strategies for tissue engineering of whole organs. Front Bioeng Biotechnol. 2015;3(March):1-17. doi:10.3389/fbioe.2015.00043. 
24. Bader A, Macchiarini P. Moving towards in situ tracheal regeneration: the bionic tissue engineered transplantation approach. J Cell Mol Med. 2010;14(7):1877-1889. doi:10.1111/j.1582-

4934.2010.01073.x.

25. Nichols JE, Niles JA, Cortiella J. Design and development of tissue engineered lung: Progress and challenges. Organogenesis. 2009;5(2):57-61. doi:10.4161/org.5.2.8564.

26. Jungebluth P, Go T, Asnaghi A, et al. Structural and morphologic evaluation of a novel detergentenzymatic tissue-engineered tracheal tubular matrix. J Thorac Cardiovasc Surg. 2009;138(3):586-593; discussion 592-593. doi:10.1016/j.jtcvs.2008.09.085.

27. Hinderer S, Schenke-Layland K. Tracheal tissue engineering: building on a strong foundation. Expert Rev Med Devices. 2013;10(1):33-35. doi:10.1586/erd.12.74.

28. Gonfiotti A, Jaus MO, Barale D, et al. The first tissue-engineered airway transplantation: 5-year followup results. Lancet (London, England). 2014;383(9913):238-244. doi:10.1016/S0140-6736(13)62033-4.

29. Badylak SF, Weiss DJ, Caplan A, Macchiarini P. Engineered whole organs and complex tissues. Lancet (London, England). 2012;379(9819):943-952. doi:10.1016/S0140-6736(12)60073-7.

30. Omori K, Tada Y, Suzuki T, et al. Clinical application of in situ tissue engineering using a scaffolding technique for reconstruction of the larynx and trachea. Ann Otol Rhinol Laryngol. 2008;117(9):673-678. http://www.ncbi.nlm.nih.gov/pubmed/18834070. Accessed January 10, 2016.

31. Jungebluth P, Alici E, Baiguera S, et al. Tracheobronchial transplantation with a stem-cell-seeded bioartificial nanocomposite: a proof-of-concept study. Lancet. 2011;378(9808):1997-2004. doi:10.1016/S0140-6736(11)61715-7.

32. Seifalian AM, Salacinski HJ, Tiwari A, Edwards A, Bowald S, Hamilton G. In vivo biostability of a poly(carbonate-urea)urethane graft. Biomaterials. 2003;24(14):2549-2557. doi:10.1016/S01429612(02)00608-7.

33. Grillo HC. Development of tracheal surgery: a historical review. Part 2: treatment of tracheal diseases. Ann Thorac Surg. 2003;75(3):1039-1047. doi:10.1016/S0003-4975(02)04109-7.

34. Göpferich A. Mechanisms of polymer degradation and erosion. Biomaterials. 1996;17(2):103-114. http://www.ncbi.nlm.nih.gov/pubmed/8624387. Accessed January 11, 2016.

35. Gunatillake P a., Adhikari R, Gadegaard N. Biodegradable synthetic polymers for tissue engineering. Eur Cells Mater. 2003;5:1-16. doi:vol005a01 [pii].

36. Freed LE, Vunjak-Novakovic G, Biron RJ, et al. Biodegradable polymer scaffolds for tissue engineering. Nat Biotechnol. 1994;12(7):689-693. doi:10.1038/nbt0794-689.

37. Middleton JC, Tipton AJ. Synthetic biodegradable polymers as orthopedic devices. Biomaterials. 2000;21(23):2335-2346. http://www.ncbi.nlm.nih.gov/pubmed/11055281. Accessed November 10, 2015.

38. Martina M, Hutmacher DW. Biodegradable polymers applied in tissue engineering research: a review. Polym Int. 2007;56(2):145-157. doi:10.1002/pi.2108.

39. Melchiorri AJ, Hibino N, Best CA, et al. 3D-Printed Biodegradable Polymeric Vascular Grafts. Adv Healthc Mater. 2015. doi:10.1002/adhm.201500725.

40. Armentano I, Dottori M, Fortunati E, Mattioli S, Kenny JM. Biodegradable polymer matrix nanocomposites for tissue engineering: A review. Polym Degrad Stab. 2010;95(11):2126-2146. doi:10.1016/j.polymdegradstab.2010.06.007.

41. Lischke R, Pozniak J, Vondrys D, Elliott MJ. Novel biodegradable stents in the treatment of bronchial stenosis after lung transplantation. Eur J Cardiothorac Surg. 2011;40(3):619-624. doi:10.1016/j.ejcts.2010.12.047.

42. Fishman JM, Lowdell M, Birchall MA. Stem cell-based organ replacements-Airway and lung tissue engineering. Semin Pediatr Surg. 2014;23(3):119-126. doi:10.1053/j.sempedsurg.2014.04.002.

43. Kojima K, Bonassar LJ, Roy AK, Mizuno H, Cortiella J, Vacanti CA. A composite tissue-engineered trachea using sheep nasal chondrocyte and epithelial cells. FASEB J. 2003;17(8):823-828. doi:10.1096/fj.02-0462com.

44. Takahashi K, Yamanaka S. Induction of pluripotent stem cells from mouse embryonic and adult fibroblast cultures by defined factors. Cell. 2006;126(4):663-676. doi:10.1016/j.cell.2006.07.024.

45. Kobayashi K, Nomoto Y, Suzuki T, et al. Effect of fibroblasts on tracheal epithelial regeneration in vitro. Tissue Eng. 2006;12(9):2619-2628. doi:10.1089/ten.2006.12.2619.

46. Nomoto Y, Kobayashi K, Tada Y, Wada I, Nakamura T, Omori K. Effect of fibroblasts on epithelial regeneration on the surface of a bioengineered trachea. Ann Otol Rhinol Laryngol. 2008;117(1):59-64. http://www.ncbi.nlm.nih.gov/pubmed/18254373. Accessed January 5, 2016.

47. Mehrban N, Teoh GZ, Birchall MA. 3D bioprinting for tissue engineering: Stem cells in hydrogels. Int J Bioprinting. 2016;2. doi:10.18063/IJB.2016.01.006.

48. Chang JW, Park SA, Park J-K, et al. Tissue-Engineered Tracheal Reconstruction Using ThreeDimensionally Printed Artificial Tracheal Graft: Preliminary Report. 2014;38(6). 
doi:10.1111/aor.12310. 